\title{
Desarrollo de barras de cereales nutritivas y efecto del procesado en la calidad proteica
}

\author{
Development of nutritive cereal bars and effect \\ of processing on the protein quality
}

\begin{abstract}
Commercial cereal bars (CB) have low and poor nutritional quality of protein and fats and are included in school snacks. A nutritional CB was developed with increased protein and fat content and quality, with a balanced energy distribution. The influence of processing on protein quality was studied. The process was divided into two stages: controlled formulation dry premix (DP) and further agglutination before consumption. In DP textured grains were used (rice, whole corn and semolina, wheat germ, oats), egg albumin and milk powder. Honey, egg white, soybean oil and sucrose were used as binding syrup. The processing was carried out in a pilot plant with drying at $105^{\circ} \mathrm{C}$, per 30 minutes. Percentage and protein quality (NPU) were determined at each stage: DP, CB before and after drying (HCB and DCB). In DCB fatty acids (FA) were determined by gas chromatography and shelf-life through sensorial evaluation by a trained panel. The DP protein content reached was $21.6 \%$ with NPU $70 \%$ and 91 $\%$ of D. For HCB and DCB the results were: protein $14.7 \%$ and $15.7 \%$, NPU $73 \%$ and $51 \%$, D $86 \%$ and $81 \%$ respectively. In DCB fat was $12.6 \%$ (16\% saturated FA, $34 \%$ monounsaturated, $48 \%$ polyunsaturated). The energy distribution was balanced. Sensory evaluation showed stable products for 7 days. It would be possible to develop CB in bakeries with short life where a unit of $25 \mathrm{~g}$ would cover $7 \%$ of the protein requirement of a $30 \mathrm{~kg}$ child. Key words: cereal bars, proteins, fatty acids, nutrition, shelf-life.
\end{abstract}

\section{INTRODUCCIÓN}

En la formulación y obtención de barras de cereales (BC), han trabajado distintos grupos de investigación en los últimos años, incorporando ingredientes autóctonos como el algarrobo (1), las avellanas (2), y en algunos casos mejorando su calidad nutricional $(3,4)$. Sin embargo, el desarrollo de BC comerciales se ha focalizado fundamentalmente en la obtención de productos de buenas características tecnológicas y organolépticas, prolongada vida útil a temperatura ambiente y formulación en base a ingredientes de bajo costo.

Trabajos recientes muestran que en la mayoría de los productos comerciales el promedio del contenido de proteínas es de tan sólo 5,5\%, y su calidad proteica sería pobre en la medida que provienen principalmente de cereales (arroz, avena, maíz) $(5,6)$. Las grasas utilizadas se encuentran lejos de las recomendaciones actuales, con ausencia de ácidos grasos poliinsaturados y frecuente presencia de grasas saturadas $y / 0$ aceites vegetales hidrogenados $(5,6)$. Éstos últimos pueden aportar en algunos casos, ácidos grasos trans cuyos niveles en
Margarita Olivera C. (1)

Verónica Ferreyra D. (2)

Silvia Giacomino M. (1)

Ana Curia C. (2)

Néstor Pellegrino G. (1)

Martín Fournier U. (2)

Nicolás Apro C. (2)

1) Cátedra de Bromatología, Facultad de Farmacia y Bioquímica, Universidad de Buenos Aires. Buenos Aires, Argentina. (2) Instituto Nacional de Tecnología Industrial (INTI), Centro de Cereales y Oleaginosas. Ciudad 9 de Julio, Provincia de Buenos Aires, Argentina.

Dirigir la correspondencia a:

M. Olivera Carrión Cátedra de Bromatología Facultad de Farmacia y Bioquímica Universidad de Buenos Aires

Junín 956, $2^{\circ}$ piso, CP 1113, Buenos Aires, Argentina. Telefono: $00541149648242 / 3$ E-mail: molivera@ffyb.uba.an

Este trabajo fue recibido el 6 de Marzo de 2012 y aceptado para ser publicado el 1 de Junio de 2012. una sola unidad de BC pueden cubrir un elevado porcentaje del máximo admisible establecido por OMS para la dieta: $1 \%$ de la energía total (7). Este aspecto deberá cambiar en breve, ya que las grasas de los alimentos no deberán contener más del $5 \%$ de grasas trans, de acuerdo a la reglamentación nacional aprobada el año 2010 es incorporada en el Artículo 155 tris del Código Alimentario Argentino (8). La tendencia por el momento es reemplazar gran parte de las grasas hidrogenadas por aceite de girasol alto oleico o mantecas vegetales ricas en grasas saturadas, sin incorporar poliinsaturadas (7).

En consecuencia, a pesar del posicionamiento como alimentos saludables (9-11), su gran difusión y vertiginoso aumento de la producción a nivel mundial (12), las BC comerciales están lejos de responder a la calidad nutricional que potencialmente podrían presentar (13).

También es conveniente considerar que las BC han sido incorporadas en algunos programas de asistencia alimentaria de comedores escolares, tanto en desayunos como en colaciones, con el objetivo de incluir productos de grano entero 
y diversificar la escasa oferta de productos de colación para escolares. Estas BC podrían y deberían presentar mejor calidad nutricional que las actualmente presentes en el mercado.

En el desarrollo de formulaciones más saludables, es necesario considerar que el proceso de elaboración comprende una etapa de mezclado de los ingredientes secos, aglutinación con grasas/azúcares, y posterior secado. Las condiciones de éste último paso son muy diferentes, variando los rangos de tiempos y temperaturas desde 50 minutos a $60^{\circ} \mathrm{C}$ hasta 45 minutos a $120^{\circ} \mathrm{C}(1,2)$.

Durante la etapa de calentamiento podrían desarrollarse reacciones de pardeamiento no enzimático como la Reacción de Maillard, debido a la presencia de azúcares junto a proteínas (14). Sin embargo, no se encontraron estudios de la influencia del proceso de obtención de BC sobre la calidad proteica, siendo esto necesario sobre todo en productos con elevada aceptabilidad en escolares (15).

Por otro lado, las BC son productos que pueden adecuarse a la mayoría de las metas de la OMS para dieta saludable: sustituir las grasas saturadas por insaturadas, eliminar los ácidos grasos (AG) trans, aumentar el consumo de granos enteros, legumbres y frutos secos (16). También es necesario considerar que actualmente se recomienda no solo limitar o promover algunos componentes aislados, sino considerar al alimento en su totalidad de acuerdo al concepto de perfil nutricional, sobre todo en productos formulados $(17,18)$.

El objetivo del presente trabajo fue estudiar la influencia del proceso de elaboración teniendo en cuenta el posible deterioro proteico durante la aglutinación y secado en pre- sencia de azúcares. En la formulación se contempló aumentar la cantidad y calidad de proteínas, mejorar el perfil de AG empleando fuentes de grasas alternativas a las utilizadas en los productos comerciales y aplicar pautas de distribución energética equilibrada de macronutrientes de acuerdo a las recomendaciones actuales para productos saludables $(16,17)$.

También se buscó unir los objetivos nutricionales con la posibilidad de dividir la elaboración de BC en dos etapas bien diferenciadas: 1) obtención en industrias o molinos de una "premezcla seca" de formulación controlada; 2) aglutinación posterior utilizando aceites vegetales ricos en AG poliinsaturados, en panaderías o empresas pequeñas de panificación, con obtención de productos de corta vida útil previo a la comercialización en los mismos establecimientos.

\section{MATERIALES Y MÉTODO}

La extrusión de los ingredientes, así como la obtención del aceite de soja y la elaboración de las muestras se realizaron en la Planta Piloto del Centro Cereales y Oleaginosas del Instituto Nacional de Tecnología Industrial (INTI), en la Ciudad de 9 de Julio, Provincia de Buenos Aires. Se trabajó a escala semiindustrial con distintas condiciones de mezclado, tiempo y temperatura, hasta la obtención de un producto de buenas características organolépticas adaptando el proceso según Ferreyra (19), según se detalla más adelante.

Formulación y elaboración. El diagrama de flujo que resume las etapas del proceso de elaboración se presenta en figura 1.

La proporción de ingredientes secos (PS), representó 60\%

FIGURA 1

Diagrama de flujo del proceso de elaboración de las barras de cereales

Ingredientes secos (PS) $60 \%$ + aglutinante $40 \%$

Mezclado $10 \min$ a $80^{\circ} \mathrm{C}$<smiles>[13CH3]I</smiles>

Formado de bandejas

Precortado con molde

$\mathrm{BCH}$ : Barra de cereal húmeda

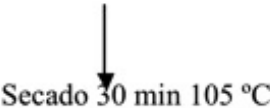

Enfrlamiento

temperatura ambiente

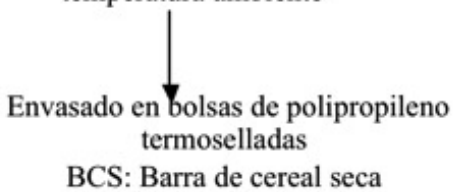


de la formulación de la mezcla húmeda. Las materias primas utilizadas fueron: 1) de origen vegetal: expandido de arroz, expandido de sémola de maíz, maíz entero extrudido, avena arrollada, germen de trigo; 2 ) de origen animal: ovoalbúmina y leche entera deshidratada.

La proporción del aglutinante fue de $40 \%$ de la mezcla húmeda. Se utilizó miel, clara de huevo, aceite de soja y sacarosa (azúcar común). El aceite de soja utilizado fue extraído por presión en frío y sin refinar.

Para la elaboración, se agregó a la premezcla seca (PS), el aglutinante a $80^{\circ} \mathrm{C}$, mezclando hasta su homogeinización. Se distribuyó uniformemente en bandejas con espesor de $1 \mathrm{~cm}$ y se precortó con molde, obteniendo así las barras de cereal húmeda $(\mathrm{BCH})$.

Las condiciones de secado fueron $105^{\circ} \mathrm{C}$ durante 30 minutos. Las barras de cereal seca (BCS) se enfriaron a temperatura ambiente y se envasaron en bolsitas de polipropileno de baja densidad termoselladas en paquetes de 4 unidades.

Metodología analítica. Las determinaciones de humedad, cenizas, proteínas, grasas, fibra dietaria, carbohidratos, valor energético, calidad y digestibilidad de proteínas y composición en ácidos grasos se realizó según la metodología detallada.

La humedad se determinó por secado hasta peso constante a $100-105^{\circ} \mathrm{C}$ de acuerdo al método AOAC 925.09 (20). La determinación de grasa se realizó aplicando hidrólisis ácida según método AOAC 922.06 (20). Las cenizas se determinaron en mufla a $550{ }^{\circ} \mathrm{C}$ hasta peso constante según método AOAC 923.03 (20). Para el contenido de proteínas se aplicó el método de Kjeldahl AOAC 984.13 (21), utilizando 6,25 como factor de conversión de nitrógeno a proteínas en todas las muestras. La fibra dietaria se determinó según el método enzimático-gravimétrico AOAC 985.29 (21). Los carbohidratos se calcularon por diferencia.

La determinación del perfil de ácidos grasos se realizó mediante cromatografía gaseosa en un Cromatógrafo Perkin Elmer Claurus 500 equipado con un detector de ionización de llama. La materia grasa se derivatizó según Norma IRAM 5650 Parte II (22) y los ácidos grasos fueron separados utilizando una columna capilar SUPELCO SP 2560 de 100 metros de largo. Los ácidos grasos fueron identificados contra patrones SUPELCO F.A.M.E. Mix NHI-C y F.A.M.E. Mix C14-C22.

Todos los análisis se realizaron por triplicado y los resul- tados se expresaron en g por $100 \mathrm{~g}$ de muestra.

La distribución energética se determinó en BCS en base a su composición centesimal utilizando los factores de Atwater.

Ensayos biológicos. De acuerdo a Frías y col (23), se determinó Utilización Proteica Neta (UPN) y Digestibilidad (D) y se calculó el Valor Biológico (VB) que resulta del cociente entre UPN y D.

A efectos de realizar los ensayos en forma simultánea y dada la capacidad del bioterio y la cantidad total de animales del ensayo (60 ratas), se seleccionaron las siguientes muestras: ovoalbúmina, PS, BCH, BCS y el producto de otra elaboración $\left(B C S^{*}\right)$. La ovoalbúmina deshidratada se estudió por ser el principal ingrediente aportador de proteínas de buena calidad nutricional. $\mathrm{BCS}^{*}$ se incluyó a efectos de estimar la influencia de distintas elaboraciones a escala artesanal en panaderías.

De cada muestra se formularon dietas experimentales al $10 \%$ de proteínas siendo la única fuente de nitrógeno la muestra en estudio, en simultáneo a una dieta de referencia al $10 \%$ de caseína suplementada con L-metionina y una dieta libre de proteínas. Para cada una de las dietas en estudio se utilizó un lote de 10 ratas de la cepa Wistar (50-60 g) de cualquier sexo y durante la experiencia los animales tuvieron libre acceso al consumo de agua y alimento, registrándose ingesta y evolución del peso corporal cada 48 horas. Los ensayos se realizaron en el bioterio existente en la Cátedra de Bromatología de la Facultad de Farmacia y Bioquímica - UBA.

Vida útil. Se estudió mediante un panel de 6 evaluadores seleccionados mediante la norma ISO 8586 (24), realizándose una totalidad de 6 sesiones de entrenamiento. La instrucción se realizó en la característica (descriptor) de aroma/sabor (flavor) rancio-oxidado, según norma AOCS (25). Se utilizó una escala de 0 a 10 puntos (figura 2). La metodología sensorial de entrenamiento y medición empleada en el ensayo estuvo basada en el análisis descriptivo cuantitativo QDA (26).

En el envasado de las muestras la condición de hermeticidad no fue contemplada a efectos de reproducir condiciones similares al proceso en panaderías desde la elaboración hasta la venta. Se diseñó un sistema escalonado de almacenamiento a $40 \pm 2^{\circ} \mathrm{C}$ durante $0,3,4,7,9$ y 11 días, tomando las muestras para cada tiempo como una unidad experimental $(26,27)$. La muestra 0 días se consideró la muestra control o muestra fresca y se almacenó a $-18^{\circ} \mathrm{C}$ durante la totalidad del tiempo de almacenamiento. La muestra 11 días fue la primera en ser

\section{Análisis Descriptivo de Barras de Cereales}

Evaluador:

Fecha:

Muestra:

Intensidad de rancio-oxidado

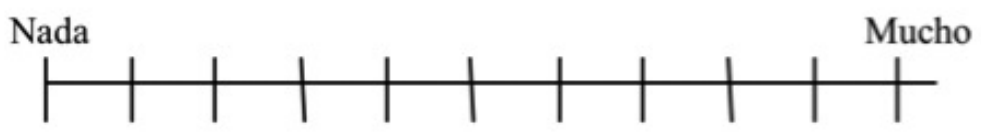


colocada en estufa de secado calibrada a $40^{\circ} \mathrm{C}$; el resto de las muestras permanecieron a $-18^{\circ} \mathrm{C}$ para inhibir el deterioro hasta el momento que debían ser introducidas a $40^{\circ} \mathrm{C}$. Al cumplir los 11 días, todas las unidades experimentales fueron retiradas de la condición de almacenamiento 4 horas antes de la sesión de medición por el panel entrenado. Se realizaron 2 sesiones de medición en las cuales las 6 unidades experimentales se evaluaron simultáneamente y por duplicado.

Se presentaron las muestras en orden al azar, en pequeñas porciones de aproximadamente $10 \mathrm{~g}$, servidas en vasitos descartables blancos de $70 \mathrm{ml}$ codificados con números de 3 dígitos aleatorios. Durante el entrenamiento como en la medición de las $B C$ se empleó agua mineral a $27^{\circ} \mathrm{C}$ y manzana Red Delicious como neutralizante entre muestras.

Análisis estadístico. Los datos analíticos de composición fueron expresados como la media \pm desviación estándar (DE) de tres determinaciones independientes. Los resultados de los ensayos biológicos (UPN y Valor Biológico), fueron expresados como la media \pm DE de 10 determinaciones independientes, aplicando análisis de Varianza (ANOVA) y test de comparaciones múltiples (Student-Newman-Keuls).

\section{RESULTADOS}

Los productos obtenidos presentaron buen aspecto con color dorado y aroma agradable.

Se analizó la premezcla de ingredientes secos (PS), luego del agregado del aglutinante (barra de cereal húmeda, $\mathrm{BCH}$ ) y posteriormente al secado (barra de cereal seca BCS).

Respecto a los ingredientes, se analizaron sólo aquellos que podrían presentar variaciones en algún parámetro (por ejemplo contenido de proteínas), según su método de obtención (ovoalbúmina deshidratada y germen de trigo extrudido). El resto de las materias primas (expandido de maíz, de arroz, avena laminada), se analizaron y chequearon previamente, comprobando que presentaban valores promedios característicos a su variedad tanto en composición como en calidad proteica y perfil de AG (28).

Composición. La humedad y datos de composición de ingredientes seleccionados y las muestras en las distintas etapas del proceso, se presentan en tabla 1.

Los valores de humedad antes y después del secado, muestran que el contenido de agua disminuye aproximadamente a la mitad, encontrándose en los niveles del 7,6\%.

Las proteínas se encontraron en el orden de $21,6 \%$ en PS y $15,7 \%$ en BCS, mientras que el contenido graso fue de $12,6 \%$ para BCS, alcanzándose los niveles iniciales propuestos. El producto desarrollado presentó buen aporte calórico, con un valor energético de 404 kilocalorías cada 100 g.

Calidad proteica. En la tabla 2 se presentan los resultados obtenidos de los ensayos biológicos para la calidad proteica total mediante la Utilización Proteica Neta (UPN), la digestibilidad (D) y el Valor Biológico (VB).

La ovoalbúmina, por su porcentaje proteico (85\%), sería el principal ingrediente condicionante del contenido y la calidad

\section{TABLA 1}

Datos de composición de ovoalbúmina, germen de trigo y muestras de barras de cereales en distintas etapas de elaboración.

\begin{tabular}{|c|c|c|c|c|c|}
\hline $\begin{array}{l}\text { DETERMINACIONES }{ }^{1} \\
\mathrm{~g} / 100 \mathrm{~g}\end{array}$ & Ovoalbúmina & Germen de trigo & PS & $\mathrm{BCH}$ & $\mathrm{BCS}$ \\
\hline HUMEDAD & $6.3 \pm 0.2$ & $7.4 \pm 0.1$ & $8.9 \pm 0.2$ & $18.5 \pm 0.1$ & $9.9 \pm 0.3$ \\
\hline CENIZAS & $5.48 \pm 0.01$ & $4.54 \pm 0.01$ & $2.10 \pm 0,04$ & $1.35 \pm 0.03$ & $1.46 \pm 0.02$ \\
\hline PROTEINAS* & $85.0 \pm 0.2$ & $34.1 \pm 0.2$ & $21.6 \pm 0.1$ & $14.7 \pm 0.1$ & $15.7 \pm 0.1$ \\
\hline GRASAS & & $13.6 \pm 0.2$ & $6.8 \pm 0.2$ & $10.1 \pm 0.1$ & $12.6 \pm 0.1$ \\
\hline FIBRA DIETARIA & & $8.4 \pm 0.1$ & $4.6 \pm 0.1$ & - & $3.4 \pm 0.2$ \\
\hline \multicolumn{6}{|l|}{ CARBOHIDRATOS } \\
\hline (por diferencia) & & 32 & 56 & - & 57 \\
\hline VALOR ENERGÉTICO Kcal & & 418 & 373 & - & 404 \\
\hline
\end{tabular}

${ }^{1}$ Valores expresados como la media de 3 determinaciones \pm SD.

PS: premezcla seca; BCH: barra cereal húmeda ; BCS: barra cereal seca; *factor 6,25

TABLA 2

Calidad nutricional proteica de ovoalbúmina y de barras de cereales en las distintas etapas de la elaboración.

\begin{tabular}{|c|c|c|c|c|c|c|}
\hline Muestras & $C M$ & Ovoalbúmina & PS & $\mathrm{BCH}$ & $\mathrm{BCS}$ & $\mathrm{BCS}^{*}$ \\
\hline UPN & $81,4 \pm 4,3$ & $75,6 \pm 6,8$ & $69,6 \pm 4,7$ & $72,9 \pm 3,9^{a}$ & $50,7 \pm 4,7^{b}$ & $49,1 \pm 5,5^{b}$ \\
\hline Digestibilidad & 96,5 & 96,2 & 91,6 & 86,2 & 80,7 & 81,5 \\
\hline Valor biológico & $84,4 \pm 4,4$ & $78,6 \pm 7,0$ & $76,0 \pm 5,1$ & $84,5 \pm 4,5^{a}$ & $62,9 \pm 5,9^{b}$ & $60,3 \pm 6,8^{b}$ \\
\hline
\end{tabular}


proteica del producto final, debido a lo cual se le realizaron los ensayos biológicos. Los valores obtenidos fueron elevados tanto para el UPN (76) como para la D (96), confirmando los valores esperables para dicho ingrediente y la buena calidad del producto utilizado.

En PS también se obtuvieron altos niveles para el UPN (70\%) y la D (91\%), lo cual indicaría que se alcanzó uno de los objetivos propuestos de complementación de las proteínas de origen vegetal con la ovoalbúmina.

Cuando se compararon los valores obtenidos antes y después del secado (BCH y BCS), se observó que existen diferencias significativas con fuerte disminución en el UPN: $73 \%$ y $51 \%$ respectivamente. La digestibilidad también disminuye, aunque no en forma tan acentuada: $86 \%$ y $81 \%$. En cambio, no se observaron diferencias significativas en ninguno de los parámetros entre las distintas elaboraciones (BCS y BCS*).

Calidad de lípidos. El contenido de AG expresados como esteres metílicos por $100 \mathrm{~g}$ de grasa se presentan en tabla 3. En BCS los AG saturados se encuentran en el orden de $16 \%$, los monoinsaturados $34 \%$ y poliinsaturados $48 \%$. La relación de AG omega 3 : omega 6 fue de 1:10 en PS y en el aceite, pero disminuye en el producto final, no alcanzando el rango recomendado por OMS (entre 1:5 y 1:10).

Distribución energética. En la figura 3 se observa que el aporte calórico de las proteínas se encuentra en el orden del 15\% (P\%), las grasas en el 26\% (F\%), siendo el resto de la energía metabolizable (58\%), proporcionado por los carbohidratos (CH\%).

Vida útil. Se seleccionó la variable aroma/sabor ranciooxidado, ya que durante las pruebas de desarrollo de las $\mathrm{BC}$ se observó que éste era el descriptor crítico que surge a través del tiempo en condiciones de almacenamiento entre $20-30^{\circ} \mathrm{C}$. El ensayo se diseñó para emplear el tiempo máximo de almacenamiento de 11 días, que sería un criterio práctico realizable en panaderías. La condición de temperatura de estudio seleccionada fue de $40^{\circ} \mathrm{C}$, ya que es normalmente la máxima registrada en Argentina durante la estación de verano.

Para la determinación de la vida útil se utilizó el método de punto de corte (29). Se graficó una regresión polinomial de segundo orden que demostró ser la más eficiente en función de los valores obtenidos para las variables tiempo en días e intensidad del descriptor rancio-oxidado en escala 1-10 (figura 4). El punto de corte obtenido fue 3,6, valor que refleja el mínimo cambio perceptible por los panelistas entrenados frente a la aparición del sabor/aroma rancio oxidado. De acuerdo a la curva, el valor de vida útil podría ser fijado como mínimo en 7 días, ya que las temperaturas usuales en las distintas regiones del país son inferiores a las del estudio.

\section{DISCUSIÓN}

Si bien en la formulación del producto se utilizaron los cereales básicos que se emplean en la elaboración industrial de la mayoría de las BC (avena, arroz, maíz), también se agregaron otras materias primas que realizaron un importante aporte de nutrientes. En PS se agregó ovoalbúmina y leche deshidratada, mientras que en el aglutinante se utilizó miel, germen de trigo, aceite de soja y clara de huevo.

Los ingredientes de origen vegetal fueron extrudidos con el objetivo de mejorar su textura y diversificar sus presentaciones (expandidos, texturizados), así como aumentar su estabilidad a la oxidación por inactivación de enzimas. Este aspecto era de importancia para la estabilidad de las materias primas utilizadas, ya que si bien no se esperaba obtener BC de prolongada vida útil debido a la incorporación de grasas insaturadas durante la aglutinación, era importante disponer de ingredientes que solos o formando parte de la premezcla (PS), fueran estables durante el almacenamiento en molinos y panaderías.

Como principal fuente de proteínas de buena calidad nutricional se utilizó ovoalbúmina, ya que su alto contenido en lisina permitiría la posible complementación de las proteínas de cereales deficientes en este aminoácido esencial. La utilización del producto en forma deshidratada en la premezcla seca, permitiría controlar el agregado en las cantidades adecuadas. También se facilitaría la manipulación y se eliminaría un factor de alto riesgo microbiológico, ya que el proceso de deshidratación de la ovoalbúmina se realiza luego de su pasteurización.

El contenido proteico alcanzado en el orden del $15 \%$, fue muy superior al promedio de 5,5\% de las BC comerciales (5). Los resultados confirman una importante disminución de la calidad nutricional durante el proceso de secado, a pesar de lo cual el consumo de una unidad de BC cubriría un porcentaje apreciable de los requerimientos proteicos de un escolar. Según las recomendaciones actuales FAO, un escolar de 10 años con un peso aproximado de $30 \mathrm{~kg}$, debería consumir $0,91 \mathrm{~g} / \mathrm{kg} / \mathrm{día}$ (30), equivalente $27,3 \mathrm{~g}$ diarios. Una unidad de BC de 25 g aportaría 3,75 g de proteína y considerando un UPN de 50, cubriría el 7\% de las necesidades de proteína completa del niño.

Por otro lado, como se dijo anteriormente, si bien las

TABLA 3

Ácidos grados de germen de trigo, aceite de soja y de barras de cereales en las distintas etapas de la elaboración.

\begin{tabular}{|c|c|c|c|c|c|}
\hline $\begin{array}{l}\text { Ácidos grasos } \\
\text { g / } 100 \text { g grasa }^{1}\end{array}$ & $\begin{array}{l}\text { Germen } \\
\text { trigo }\end{array}$ & $\begin{array}{l}\text { Aceite } \\
\text { de soja }\end{array}$ & PS & BCS & $\mathrm{BCS}^{*}$ \\
\hline Saturados & $19,9 \pm 0,3$ & $15,4 \pm 0,2$ & $32,0 \pm 0,8$ & $16,1 \pm 0,3$ & $16,7 \pm 0,2$ \\
\hline Monoinsaturados & $18,0 \pm 0,1$ & $21,9 \pm 0,1$ & $25,8 \pm 0,2$ & $34,2 \pm 0,2$ & $30,5 \pm 0,1$ \\
\hline Poliinsaturados & $61,1 \pm 0,3$ & $62,7 \pm 0,1$ & $40,5 \pm 0,2$ & $48,4 \pm 0,2$ & $51,4 \pm 0,2$ \\
\hline omega6 & $53,6 \pm 0,3$ & $54,8 \pm 0,1$ & $37,3 \pm 0,1$ & $47,4 \pm 0,02$ & $50,3 \pm 0,2$ \\
\hline omega3 & $5,5 \pm 0,1$ & $5,5 \pm 0,1$ & $3,1 \pm 0,1$ & $0,9 \pm 0,01$ & $1,1 \pm 0,01$ \\
\hline Omega 3 : Omega 6 & $1: 10$ & $1: 10$ & $0,8: 10$ & $0,2: 10$ & $0,2: 10$ \\
\hline
\end{tabular}

${ }^{1}$ Valores de los ésteres metílicos en $100 \mathrm{~g}$ expresados como la media de 3 determinaciones $\pm \mathrm{DE}$.

PS: premezcla seca; $\mathrm{BCH}$ : barra cereal húmeda; $\mathrm{BCS}$ : barra cereal seca; $\mathrm{BCS}{ }^{*}$ : barra cereal seca de diferente elaboración. 
FIGURA 3

Comparación de la distribución energética de los macronutrientes en barras de cereales seca con las recomendaciones de la OMS.

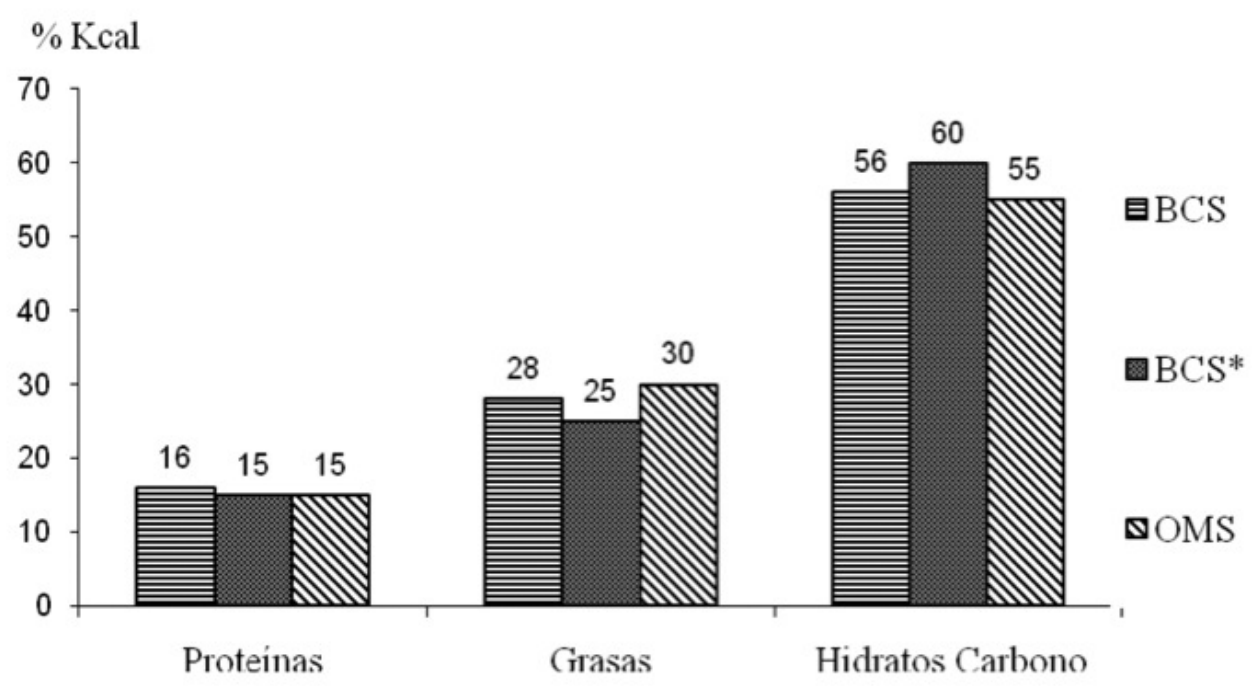

BCS: barra cereal seca; BCS*: barra cereal seca de diferente elaboración.

FIGURA 4

Vida útil de barras de cereales a $40{ }^{\circ} \mathrm{C}$ determinado por panel entrenado mediante evaluación sensorial

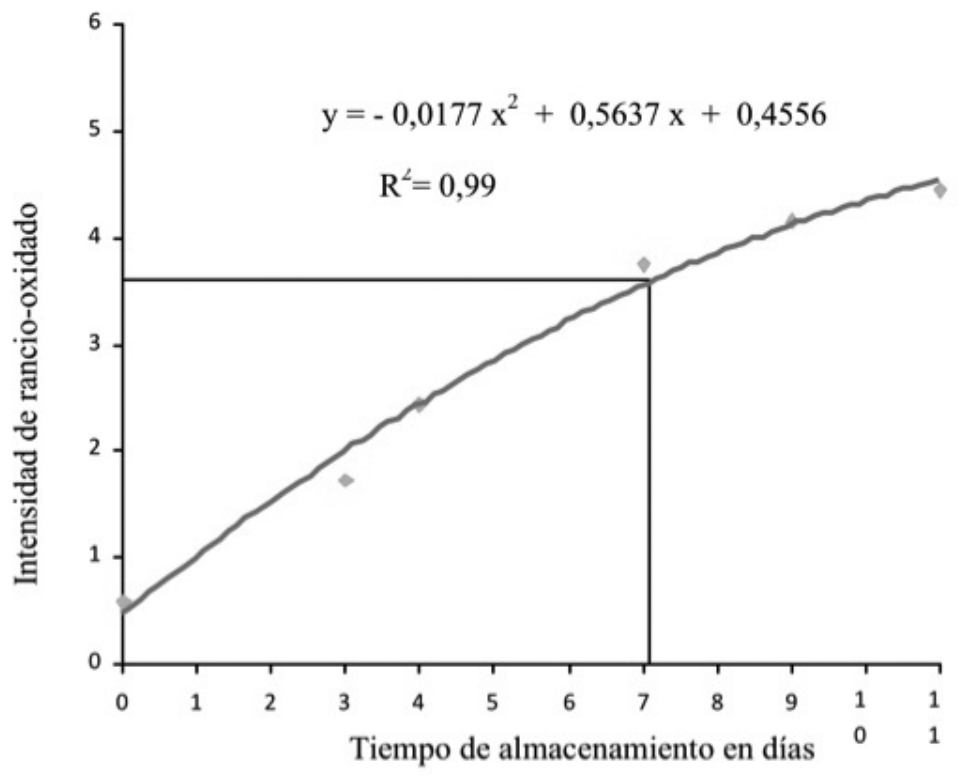


grasas y azúcares son necesarios para una correcta aglutinación, la composición del aglutinante en el nuevo producto. fue muy diferente a los comerciales. Industrialmente los azúcares empleados son en general jarabes de maíz, glucosa o azúcar, en proporciones relativas importantes ya que constituyen el primer y/o segundo ingrediente en el 56 y $50 \%$ de los casos (5). En cambio, en el producto desarrollado, la miel constituyó la principal fuente de azúcares, agregándose sacarosa en pequeñas proporciones, ya que por razones organolépticas era conveniente disminuir la predominancia de la miel. Por otro lado, las cantidades utilizadas fueron las compatibles con las necesidades tecnológicas y un sabor ligeramente dulce.

Respecto a las grasas, estas son aportadas principalmente por aceite de soja y el germen de trigo, siendo minoritario el aporte de la leche entera. Si bien no fue posible alcanzar la relación AG omega 3: omega 6 deseable, fue factible la aglutinación utilizando aceites vegetales ricos en AG poliinsaturados. El perfil de AG obtenido se diferencia netamente de los productos comerciales, ya que en éstos predominan AG oleico, saturados y/o trans según se utilice aceites de girasol alto oleico, mantecas vegetales y/o aceites vegetales hidrogenados respectivamente (7). Las $B C$ comerciales no proporcionan AG poliinsaturados, ya que se prioriza la estabilidad al deterioro oxidativo de los AG saturados y trans, a efectos de conservar los productos estables durante toda su vida útil, en general de un año (5).

La distribución energética alcanzada de los macronutrientes (E\%) fue equilibrada acorde a las recomendaciones actuales para los productos formulados (16-18). Este perfil nutricional se diferenció claramente del obtenido en BC comerciales, donde según distintos investigadores, la energía es proporcionada por carbohidratos en niveles del $80 \%$ (6) y la grasa total supera el $30 \%$ de la energía en la tercera parte de la muestras (7).

El producto obtenido, de acuerdo a resultados previos, obtuvo muy buena aceptación sensorial en escolares de distintos niveles socioeconómicos (15), lo cual permitiría su utilización en programas alimentarios para escuelas reemplazando a las actualmente utilizadas.

Por otro lado, se acercaría al concepto de producto natural que tienen las barras de cereales, originado en la mezcla de cereales, leche y miel, en formato de "alimento en barra" para facilitar su consumo y hacerlo compatible con el ritmo de la vida urbana actual.

\section{CONCLUSIONES}

Fue posible la formulación de BC de elevado contenido proteico y a pesar de la disminución significativa de la calidad proteica inherente al secado, una unidad de BC cubriría un porcentaje apreciable de los requerimientos proteicos de un escolar. En la aglutinación fue posible utilizar aceites vegetales con alto grado de insaturación compatible con productos de corta vida útil. Los productos cumplen además con una buena distribución energética de los macronutrientes.

Las BC desarrolladas ofrecerían una alternativa de alimentos saludables para diversificar la pobre oferta de productos de colación. Sería posible la elaboración en etapas, con premezclas de composición controlada y aglutinación en pequeños emprendimientos de panificación o panaderías previo a la venta.

\section{RESUMEN}

Las barras de cereales (BC) comerciales presentan bajo contenido y pobre calidad nutricional de proteínas y grasas, siendo actualmente incluidas en colaciones escolares. Se de- sarrollaron BC nutritivas con aumento del contenido y calidad de proteínas y grasas, distribución energética equilibrada y se estudió la influencia del procesado en la calidad proteica. El proceso se dividió en dos etapas: una premezcla seca (PS) de formulación controlada y posterior aglutinación previo al consumo. En PS se utilizaron cereales texturizados (arroz, maíz entero y sémola, germen de trigo, avena), ovoalbúmina y leche ambas deshidratadas. Para la aglutinación se utilizó miel, clara de huevo, aceite de soja y sacarosa. La elaboración se realizó en planta piloto con secado a $105^{\circ} \mathrm{C}$, por 30 minutos. Se determinó el porcentaje y la calidad proteica (UPN) en cada etapa: PS, barra de cereal húmeda $(B C H)$ y seca (BCS). En la BCS se determinaron los ácidos grasos (AG) por cromatografía gaseosa y la estabilidad mediante análisis sensorial por panel entrenado. En PS se alcanzó $21,6 \%$ de proteínas con UPN 70\% y D 91\%. Para BCH y BCS los resultados fueron: proteínas $14,7 \%$ y $15,7 \%$, UPN $73 \%$ y $51 \%$, D $86 \%$ y $81 \%$, respectivamente. En BCS el contenido graso fue 12,6\% (16\% AG saturados, $34 \%$ monoinsaturados, $48 \%$ poliinsaturados). La distribución energética de macronutrientes fue equilibrada y la evaluación sensorial arrojó productos estables durante 7 días. Sería posible elaborar en panaderías BC de corta vida útil, donde una unidad de $25 \mathrm{~g}$ cubriría $7 \%$ del requerimiento proteico de un niño de $30 \mathrm{~kg}$.

Palabras clave: barras de cereales, proteínas, ácidos grasos, nutrición, vida útil.

Agradecimientos: Se agradece a la Universidad de Buenos Aries quien financió este trabajo parcialmente a través del Subsidio UBACyT 2002009010017101.

\section{BIBLIOGRAFÍA}

1. Estévez AM, Escobar BA Ugarte V. Utilización de cotiledones de algarrobo (Prosopis chilensis) en la elaboración de barras de cereales. Arch Latinoam Nut 2000; 50 (2): 148 -51.

2. Cheuquepan AM, Villarroel M, Biolley E. Elaboración y optimización de una barra funcional de avellana chilena. Proyecto Cyted 11.19. Aplicación de ingredientes funcionales en alimentación infantil y para adultos. 2004; p 153-65.

3. Villarroel Tudesca, M, Peña Fritz C. Formulación de una barra funcional de avellana con propiedades antioxidantes. Rev Chil Nutr 2004; 21 (1): 203-53.

4. Freitas DGC, Moretti RH. Barras de cereais elaboradas com proteína de soja e gérmen de trigo, características físicoquímicas e textura durante armazenamento. Arch Latinoam Nutr 2005; 55: 299-304.

5. Olivera Carrión M.; Giacomino S.M.; Pellegrino N.; Sambucetti M.E. Composición y Perfil Nutricional de Barras de Cereales Comerciales. Actualización Nutr 2009; 10(4): 275-84.

6. Barras de Cereales. Informe de desempeño. Instituto Nacional de Tecnología Industrial (INTI). Centro de Cereales y Oleaginosas. 2011. Disponible en: http://Www.inti.gob. ar/productos/pdf/barritas_cereal2011.pd) Consultado 30 setiembre 2011.

7. Giacomino S.M.; Pellegrino N.; Olivera Carrión M. Perfil Nutricional de Barras de Cereales Comerciales según distribución energética de la grasa. Aceites y Grasas 2011; 82 (I): 104-7.

8. Código Alimentario Argentino. Capítulo III: Productos alimentícios. Disponible en: http://WwW.anmat.gov.ar CODIGOA/CAA1.HTM. Consultado enero 2012

9. Iñarritu MC, Franco LV. Las barras de cereales como ali- 
mento funcional en los niños. Rev Mexicana Pediatr 2001; 68(1): 8-12.

10. Viviant V. Barras de cereal: una golosina saludable. Alimentación Latinoam 2005; 258: 40-2.

11. Olivera Carrión M. Functional Foods in Argentina, World of Food Science, 2007, Revista electrónica del Institute of Food Tecnologists, (IFT) - International Union of Food Science and Technology (IUFOsT), 2007. Disponible en: http://www.worldfoodscience.org/pdf/FF_en_ARGENTINA_1-05.pd] Consultado 30 junio 2011.

12. Winter J. Energy bars. Functional Ingredients, revista editada por Pentom Media Inc, USA, march 2008; $p$ 38-42.

13. Olivera Carrión, M. Desarrollo de Barras de Cereales de buen perfil nutricional. Heladería - Panadería Latinoam 2010; 206: 34-40.

14. Cheftel J. C., Cheftel H. Pardeamiento no enzimático. Introducción a la bioquímica y tecnología de los alimentos. Editorial Acribia, 1976; 1: 291-308.

15. Ferreyra V, Flores A, Fournier M, Aguilar V, Apro N, Giacomino $S$ y col. Estudio de la aceptabilidad en escolares de barras de cereales formuladas con ovoalbúmina, aceite de soja y miel. DIAETA 2009; 27 (126):18-25.

16. OMS. Informe Técnico 916, Dieta, Nutrición y Prevención de Enfermedades Crónicas, 2003. Disponible en: ftp.fao. org/docrep/fao/006/ac911s/ac911s00.pdf. Consultado 30 junio 2011.

17. Rayner M, Scarborough P, Stockley L. Nutrient profiles: Applicability of currently proposed model for uses in relation to promotion of food to children aged 5-10 and adults. British Heart Foundation, Department of Public Health, University of Oxford. 2005. Disponible en WwW. food.gov.uk/multimedia/pdfs/nutprofmodelforadults.pdf Consultado 30 mayo 2011.

18. López-López I.; Cofrades S; Ruiz-Capillas C; JiménezColmenero F.Design and nutritional properties of potential functional frankfurters based on lipid formulation, added seaweed and low salt content. Meat Science 2009; 83, 255-62.

19. Ferreyra V. A. Desarrollo de Barras de Cereales Nutritivas.
Tesis de la Maestría Internacional en Tecnología de los Alimentos, Universita Degli Studi Di Parma y Facultad de Agronomía Universidad de Buenos Aires, 2010.

20. Official Methods of Analysis of the A.O.A.C. 13th Edition; Washington D.C., USA Association of Official Analytical Chemists: 1980; 14.

21. Official Methods of Analysis of the AOAC International, 17th edition. Ed. Dr. W. Horwitz. Maryland. USA. 2000.

22. IRAM, Norma 5650 Parte II. Aceites y Grasas vegetales y Animales. Método rápido de preparación de ésteres metílicos de ácidos grasos, para su análisis por cromatografía en fase gaseosa. 1982.

23. Frias J, Giacomino S, Peñas E, Pellegrino N, Ferreyra $V$, Apro N, Olivera Carrión M. and Vidal-Valverde C. Assessment of the nutritional quality of raw and extruded Pisum sativum L. var. laguna seeds. LWT- Food Sci Technol 2011; 44 (5) 1303-08.

24. ISO International Standard 8586-1. Sensory Analysis General guidance for the selection, training and monitoring of assessors. Geneva: International Standards Organization 1993.

25. AOCS. Flavor panel evaluation of vegetable oils. AOCS Recommended Practice Cg 2-83, AOCS 1989.

26. Stone, H.; Sidel J.L. Sensory evaluation Practices. Edit. Elsevier Academic Press. 2004.

27. Hough, G.; Wittig, E. Introducción al análisis sensorial. Estimación de la vida útil sensorial de los alimentos. Programa CYTED, 2005; Cap. 1: p 13-14.

28. Pellegrino, N.; Giacomino, S.; Sambucetti, M.E.; Olivera Carrión, M Caracterización de Ingredientes para Alimentos Funcionales. Trabajos completos. [CD-ROM]. XI Congreso Argentino de Ciencia Tecnología de Alimentos. Buenos Aires, Argentina. 2007.

29. Curia, A.; Garitta, L.; Restrepo, P.; López, C. Metodología de punto de corte. Estimación de la vida útil sensorial de los alimentos. Programa CYTED, 2005; 5: 71-81.

30. FAO. Protein and amino acid requirements in human nutrition. Report of Joint WHO/FAO/UNU Expert consultation. WHO Technical Report Series 935, 2007. 